\section{Commentary: The evolution of extracorporeal membrane oxygenation for COVID-19: Through the eyes of the experts}

\author{
Francis D. Pagani, MD, PhD
}

The COVID-19 pandemic is an ongoing global pandemic caused by SARS-CoV-2. The World Health Organization declared a public health emergency of international concern on January 30, 2020, and a pandemic on March 11, 2020. The pandemic of COVID-19 has evolved into one of the most important health challenges of the 21 st century and has resulted in severe social and economic disruption around the world.

Similar to past experiences with global outbreaks of viral syndromes that have caused significant respiratory disease, extracorporeal membrane oxygenation (ECMO) has emerged as a viable treatment option and rescue strategy for respiratory failure not responding to optimal or conventional care. At the start of the pandemic, the use of ECMO as a rescue strategy and its outcomes remained uncertain but experience quickly evolved and ECMO has been adopted in more cases of respiratory failure from COVID-19 disease. In an expert review presented in this issue of the Journal, Dr Hayanga and colleagues ${ }^{1}$ provide a succinct overview of the important observations noted and practices adopted during the evolution of the pandemic that have provided greater insight into the selection and management of patients receiving ECMO for COVID-19 respiratory failure. These important insights include observations on how patient characteristics for those receiving ECMO have evolved during the pandemic, a diagram outlining a triage protocol for centers without ECMO to refer to an ECMO center, a table listing important

From the Department of Cardiac Surgery, University of Michigan, Ann Arbor, Mich. Disclosures: The author is a noncompensated scientific advisor for FineHeart, Inc, and CH Biomedical, Inc, and member of the Data Safety Monitoring Board for Carmat, Inc and the National Institutes of Health, PumpKIN Study.

The Journal policy requires editors and reviewers to disclose conflicts of interest and to decline handling or reviewing manuscripts for which they may have a conflict of interest. The editors and reviewers of this article have no conflicts of interest.

Received for publication Oct 23, 2021; revisions received Oct 23, 2021; accepted for publication Oct 25, 2021; available ahead of print Oct 28, 2021.

Address for reprints: Francis D. Pagani, MD, PhD, Department of Cardiac Surgery, University of Michigan, 5161 Cardiovascular Center, SPC 5864, 1500 East Medical Center Dr, Ann Arbor, MI 48109 (E-mail: fpagani@umich.edu).

J Thorac Cardiovasc Surg 2023;165:218

$0022-5223 / \$ 36.00$

Copyright (c) 2021 by The American Association for Thoracic Surgery

https://doi.org/10.1016/j.jtcvs.2021.10.036

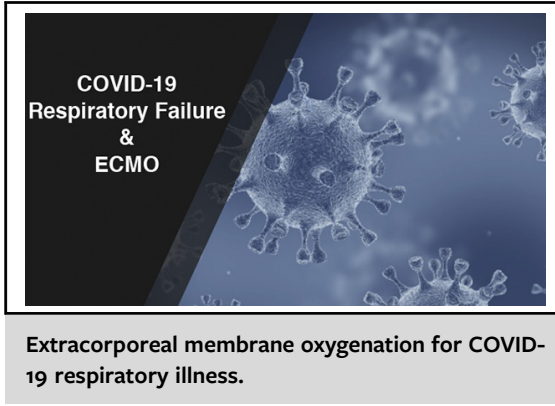

CENTRAL MESSAGE

Extracorporeal membrane oxygenation has emerged as a viable treatment alternative for respiratory failure from COVID19 disease not responding to conventional therapies.

knowledge gaps that require further investigation, pharmacological adjuncts, and ECMO procedural considerations for lung management, cannulation, and equipment necessary for ECMO support. Importantly, resource management during a pandemic, including availability of ECMO circuits, has become a critical issue, which the authors have addressed by providing insight from experiences coordinating regional ECMO resources. Comorbid conditions, particularly obesity, have been a contributing factor to respiratory failure with COVID-19 disease. The authors specifically provide consensus practices for obese patients.

As the authors point out, the evolution of the use of ECMO during the pandemic has largely occurred through the reports of nonrandomized, single-center experiences, and observations. The lack of data obtained from rigorous, prospective multicenter trials, and lack of consensus opinion has resulted in significant management variation and knowledge gaps and has been an important limitation in refining ECMO practices for COVID-19 respiratory failure. This important work by a group of recognized experts in the field of ECMO has provided an informative report on a collective experience and provides consensus opinion for the readers.

\section{Reference}

1. Hayanga JWA, Chatterjee S, Kim BS, Merritt-Genore H, Milewski RCK, Haft JW, et al. Venovenous extracorporeal membrane oxygenation in patients with COVID-19 respiratory failure. J Thorac Cardiovasc Surg. 2023;165:212-7. 